\title{
Políticas de Información: el amplio espectro de la investigación
}

\author{
Egbert J. Sánchez Vanderkast
}

Artículo recibido:

9 de julio de 2004.

Artículo aceptado: 16 de noviembre de 2004.

\section{RESUMEN}

Las políticas de información son un área de estudio de la bibliotecología y las ciencias de la información que sigue siendo poco atractiva para algunos profesionales de la bibliotecología y la información, por abarcar múltiples aristas. La complejidad del tema ha determinado el poco acercamiento al mismo por parte de los estudiosos interesados. Se pretende describir aquí algunos de los métodos de recopilación de datos propuestos por distintos autores durante los últimos veinte años. Esto con el fin de mostrar el amplio espectro de investigación que tienen las políticas de información. La recopilación sistemática de datos nos acerca a perspectivas novedosas de análisis e interpretación de las variables que intervienen en el estudio sobre políticas de información y nos ayuda a entenderlas en los distintos niveles tanto del sector público como del privado. Esto también nos servirá para realizar predicciones, explicaciones, interpretaciones, y para hacer aplicaciones, y 
generará conocimientos más profundos sobre la temática. Las aportaciones de autores de la región latinoamericana se tratarán en otra ocasión.

Palabras clave: Políticas de información; Métodos de recopilación de datos; Metodología de teoría fundada en datos.

\section{ABSTRACT}

Information policies: the ample spectrum of research Egbert J. Sánchez-Vanderkast

Library science and information studies concerned with information policies have lacked popularity among information professionals due to its multiple facets. The paper intends to describe data collection methods proposed by different authors during the last 20 years. Data gathering as a systematic procedure brings us near to novel perspectives in analysis and interpretation of variables and incidents which are part of information policy research. This may also help to understand our topic at different levels of the public and private arenas. Furthermore, it will help to promote and apply new knowledge and observe predictions and explanations on the subject.

Keywords: Information policies; Data gathering; Grounded theory.

\section{INTRODUCCIÓN}

$\mathrm{E}$ n 1997 se publicó el editorial titulado "A research agenda beyond 2000", el cual establecía una agenda de líneas de investigación para el año 2000 y en adelante en materia de políticas de información.

A ese respecto once estudiosos sobre el particular(Mary Burke, MinMin Chang, Charles Davis, Peter Hernon, Gary Marchionini, Paul Nicholls, Candy Schwartz, Debora Shaw, Alastair Smith, Stephen Wiberley, y Ann Wolpert) procedentes de diferentes universidades (USA, Canada, Irlanda, Nueva Zelanda y Kowloon, en China) emitieron sus opiniones y propusieron alrededor de veinticinco temas. En este entorno un académico se hacía las 
siguientes preguntas: ¿qué son las políticas de información? y ¿cuáles aspectos del ciclo de vida de la información tienen relación con la información gubernamental?

Por su parte, Hernon realiza otro sondeo entre los colaboradores de la revista Journal of Academic Librarianship en el mismo año, cuyos resultados fueron descritos en "Going beyond "same old, same old". De estos catorce (Nicholas Burckel, Ronald Dow, Karyle Sue Butcher, Don Frank,William Goslling, Larry Hardesty, Edely Hogan, Cerril LaGuardia, Laurie Linsley, Sarah Pritchard Carlen Ruschoff, Charles Lowry, Helen Spalding y John Sulzer), sólo dos mencionaban en relación con la temática de políticas de información "el papel del bibliotecario en la hechura de políticas nacionales de información” y las "políticas gubernamentales de información”.

Para 1998 el Government Information Quarterly realizó un foro de discusión con el propósito de considerar los tópicos relacionados con las políticas de información gubernamental. Los convocados fueron Patrick J. Birkinshaw, Gary Cornwell, Margin Farell, Robert Gellman, Jane Bortnick Griffith, Jane E Kirtley, Charles R. McClure, Harold Relyea y John Shuller, quienes propusieron 30 tópicos en los que era necesario realizar indagaciones.

Hernon, editor del Government Information Quarterly GIQ enfatizó que en el ámbito de políticas de información para el sector gubernamental había mucha investigación por hacer e incluyó la siguiente lista:

a) Análisis de la infraestructura global y nacional.

b) Eficiencia y calidad de los servicios que suministran información gubernamental.

c) Nivel de satisfacción de los servicios y prácticas en el entorno gubernamental.

d) Evaluación del impacto, resultado y ejecución tanto de las instituciones y organizaciones como de los instrumentos de medición utilizados

e) Las políticas gubernamentales y la gestión de la información.

El ámbito gubernamental maneja el trinomio disponibilidad-diseminación-acceso; el gobierno promueve la disponibilidad y la necesidad de diseminación de información y datos, y el público-ciudadano demanda el acceso a ellos.

En las discusiones y opiniones manifestadas sobre el tema políticas de información en general y en particular en el sector gubernamental, algunos de los participantes consideran que en esta temática lo importante es destacar que las "políticas de información" seguirán siendo uno de los temas de interés para la bibliotecología y los estudios de la información en los próximos años. 
Considerando la amplitud del tema anterior Bender, ${ }^{1}$ Liu, ${ }^{2}$ Chartrand ${ }^{3}$ y Milevski ${ }^{4}$ proponen una categorización del tema en nueve sub-temas que se enuncian a continuación:

1. Gestión ${ }^{5}$ gubernamental de las fuentes de información o recursos informativos.

2. Tecnologías de Información y Comunicación, TIC.

3. Telecomunicaciones y radiodifusión.

4. Comunicación internacional.

5. Divulgación, privacía, confidencialidad de los datos e información.

6. Regulación y delitos electrónicos y computacionales.

7. Propiedad intelectual.

8. Bibliotecas y archivos.

9. Diseminación de la información gubernamental.

Sin embargo cabe señalar que el tema sigue siendo poco atractivo para algunos profesionales de la bibliotecología y los estudios de la información dada la complejidad que presenta. En este sentido al hablar de políticas de información se trasladan éstas hacia las ciencias políticas y se olvida que la disciplina medular involucrada es la bibliotecología y los estudios de la información.

Los temas propuestos por los participantes en el Foro de discusión del GIQ, estudiosos de la políticas de información gubernamental, los colaboradores de la revista Journal of Academic Librarianship, y los académicos, Bender, Lui, Chartrand y Milevski conviertieron la temática en una línea de investigación compleja.

La visión de qué son y para qué sirven las políticas de información se diluye mientras no se tenga claro hacia dónde se encamina la investigación en esta materia.

Más aún en el ámbito académico se le augura un futuro promisorio a este tema por ser un campo de estudio emergente en el que hasta el momento no se ha probado ningún método de investigación.

Describiremos, pues, algunos de los métodos de recopilación de datos identificados entre 1984 y el primer semestre de 2004, y enunciaremos algunas

1 Cfr. D.R. Bender. "A strategy for international information policy", en LIBRI 43 (3): 210-231, 1993.

2 Cfr. Yin Quan Liu. "The impact of national policy on developing information infrastructure nationwide issues in P.R. China and the U.S", en LIBRI 46 (4):175-183, 1996.

$3 C f r$. R. J. Chartrand. "Legislating information policy", en Bulletin of the American Society for Information Science 12 (5):10, 1986.

4 Cfr. S. Milevski. "Information policy through public laws of the 95th-the 98th Congresses", en Proceedings of the American Society for Information Science Annual meeting, 23: 211-219, 1986 
de las ventajas que se obtienen al aplicar cualquiera de los métodos descritos de manera sistemática. En esta ocasión no se analiza la región de América Latina para conocer las propuestas de pares a este respecto.

\section{MÉTOdos de INVESTIGACióN}

La revisión de la literatura (Hernon, Rowlands, Eisenshitz, Braman) se describirá más adelante. Por el momento es oportuno plantearse la pregunta siguiente:

¿Qué se debe entender por investigación?

Para elaborar la respuesta adecuada se retoma el planteamiento de Hernon en el sentido de que es "un proceso de indagación y averiguación que tiene componentes particulares". ${ }^{6}$ Según este autor la investigación está estructurada en cinco grandes componentes:

1. La indagación reflexiva, que se integra de los siguientes rubros:

- El planteamiento del problema.

- La revisión de la literatura o bibliografía documental.

- El marco teórico.

- La estructura lógica.

- El objetivo.

- Las preguntas de la investigación.

- La hipótesis.

2. El procedimiento, en el cual hay que describir lo siguiente:

- El diseño de la investigación.

- El método de recopilación de datos.

3. Los métodos de recolección de datos:

- La recopilación.

- El procesamiento.

- El análisis.

4. La identificación del estudio: cuantitativo o cualitativo y

5. La presentación de los resultados .

Es preciso realizar un planteamiento de los problemas y encontrar un marco que se adecue a nuestra expectativa antes de abordar el fenómeno de estudio. Por un marco adecuado nos referimos a encontrar un referente que nos brinde algunas de las características sobresalientes sobre lo que deseamos estudiar. De no ser esto suficiente entonces tendríamos que elegir distintos referentes para armar un posible marco. Los marcos por lo general ayudan a definir el problema.

6 Hernon. "Components of research process", en Journal of Academic Librarianship 27 (2):81-89, 2001. 
Entman entiende por marco, (frame, framing, frameworks) a

"la selección de algunos aspectos de una realidad donde se destacan algunas de sus características en un escrito, de tal manera que fomente una definición particular, una interpretación causal, promueva una evaluación moral y/o recomiende un tratamiento" ${ }^{7}$

Los marcos tienen las siguientes características:

- El diagnostico, que intenta identificar a los elementos y los factores que han creado el problema.

- La evaluación, que permite obtener un juicio sobre los agentes que intervienen en los hechos y sus posibles efectos e impacto.

- La prescripción, que ofrece y justifica las alternativas a los problemas, fenómenos y hechos que emergieron y predice sus efectos para luego realizar una propuesta de corrección.

\section{Perspectiva de ANÁLISIS}

Ian Rowlands en su Understanding Information Policy considera que los problemas metodológicos en los estudios de políticas de información son múltiples y tienen una marcada tendencia por cinco perspectivas de análisis:

- Clasificación.

- Identificación de los asuntos (issue) y las opciones sobre política.

- Reducción de la complejidad.

- Prospectiva y construcción del escenario.

- Investigación orientada hacia los procesos de las políticas y los estudios de caso.

Cada una de estas propuestas tienen sus respectivas fortalezas y sus debilidades metodológicas.

La investigación desde una perspectiva de clasificación debe:

- Facilitar el acceso a los materiales para realizar investigación original.

- Proporcionar los instrumentos necesarios para explorar esquemas de datos complejos. munication 43 (4):51-58, 1993. 
- Demostrar la amplitud de los asuntos que pueden ser estudiados como políticas de información.

Mientras que las debilidades se inclinan principalmente hacia:

- Un limitado apuntalamiento teórico.

- Un contexto político, social e institucional confuso en donde opera la política.

- La posibilidad de una clasificación puede realizarse:

- por instituciones,

- por tipo de documentos,

- por objetivos o metas que pretende alcanzar una política, y

- por el nivel de la política: (implícito o explicito).

Esta metodología de agrupar (clustering), la ha utilizado el autor para ubicar los documentos relativos a áreas temáticas y asuntos muy particulares, y para separarlas de los asuntos considerados problemáticos, como la investigación científica y el desarrollo, el mercado de la información, la libertad y el acceso a la información gubernamental, y los aspectos legales como la privacía y la propiedad intelectual. Rowlands en "Some compass bearing for information policy orienteering" promueve un estudio donde agrupa las políticas de información en cinco grandes grupos:

1. La protección de la información que cobija la seguridad nacional, el secreto gubernamental u oficial, la privacidad sobre seguridad de la información y la protección de los datos y flujos de datos transfronteras.

2. El mercado de la información, que aborda los temas: mercado de la información, propiedad intelectual, derecho de copiado, flujos de datos transfronteras, protección de datos, radio difusión y servicio universal.

3. La radiodifusión y las telecomunicaciones, que agrupan la libertad de expresión, las telecomunicaciones, la legislación de los medios en general, el servicio universal y la radiodifusión.

4. El acceso a la información gubernamental: depósito legal, bibliotecas nacionales, políticas de información, libertad y acceso a la información, publicaciones gubernamentales, gestión de fuentes de información o recursos informativos gubernamentales, sociedad de la información.

5. La sociedad de la información y la infraestructura: la industria de la información, la infraestructura de la información y la sociedad de la información. 


\begin{tabular}{|c|c|}
\hline Tema & Sub-Tema \\
\hline 1. Protección de la información & $\begin{array}{l}\text { a) secreto gubernamental u oficial, } \\
\text { b) seguridad nacional, } \\
\text { c) protección de datos, } \\
\text { d) privacía, } \\
\text { e) seguridad de la información, } \\
\text { f) flujos de datos transfronteras, } \\
\text { g) divulgación de la información. }\end{array}$ \\
\hline 2. Mercado de la información & $\begin{array}{l}\text { a) mercado de la información, } \\
\text { b) propiedad intelectual, } \\
\text { c) derecho de copiado, } \\
\text { d) flujo de datos transfronteras, } \\
\text { e) protección de datos, } \\
\text { f) radio difusión, } \\
\text { g) servicios universales. }\end{array}$ \\
\hline 3. Radiodifusión y telecomunicaciones & $\begin{array}{l}\text { a) libertad de expresión, } \\
\text { b) telecomunicaciones, } \\
\text { c) legislación de los medios en general, } \\
\text { d) servicios universales, } \\
\text { e) radio difusión. }\end{array}$ \\
\hline 4. Acceso a la información gubernamental & $\begin{array}{l}\text { a) depósito legal, } \\
\text { b) bibliotecas nacionales, } \\
\text { c) políticas de información, } \\
\text { d) libertad y acceso a la información, } \\
\text { e) gestión de fuentes de información. }\end{array}$ \\
\hline $\begin{array}{l}\text { 5. Sociedad de la información y la } \\
\text { infraestructura de la información }\end{array}$ & $\begin{array}{l}\text { a) industria de la información, } \\
\text { b) infraestructura de la información, } \\
\text { c) sociedad de la información. }\end{array}$ \\
\hline
\end{tabular}

De esta manera cada propuesta temática debe incluir por escrito su interpretación y los sub-temas que abarca.

En otras palabras el primer paso es agrupar por temas comunes, el segundo asignar un título, el tercero describir el título asignado y su alcance, y el cuarto enunciar las temáticas que quedan comprendidas en dicho rubro.

\section{Análisis paradigmático}

Mairead Browne ${ }^{8}$, ha enfocado sus estudios hacia un acercamiento de lo que ella considera valor-crítico y valor paradigmático.

Ella coincide con los académicos y algunos colaboradores en que el campo de la investigación de políticas de información es muy amplio y que por

8 Cfr. Mairead Browne, "The field of information policy. 1. Fundamental concepts", en Journal of Information Science 23 (4):261-275, 1997. y "The field of information policy. 2. Redefining the boundaries and methodologies", en Journal of Information Science 23 (5):339351, 1997. 
ello hay que dar un salto y explorar algunas tradiciones metodológicas antes de abordar la temática y construir una propuesta. Para ello propone realizar reflexiones a partir del pensamiento positivista y no menospreciar las nuevas tendencias que nos brindan los estudiosos de las ciencias sociales. Los profesionales de la información que desean incursionar en este campo de acción de la bibliotecología y los estudios de la información no deben de perder de vista los aspectos históricos y tomar muy en cuenta esta frontera disciplinaria, y entender los marcos conceptuales en las cuales está sustentada nuestra disciplina. Esto los conducirá a cuestionar, entender y confiar en los fundamentos de la realidad y la vida cotidiana, así como la naturaleza del oficio de indagar y conocer.

Los mismos cambios generados en el mundo actual nos exigen ordenar el conjunto de metodologías y técnicas de recopilación de datos. Pero también hay que preguntarse
a. ¿qué pasa con los valores?
b. ¿cuáles son los valores del investigador? y
c. ¿qué papel juegan éstos en su investigación?

Entendiendo el sistema de valores que se está manejando se puede precisar sobre qué bases se encuentra fundamentado el paradigma de indagación. Por lo anterior hay que tomar en cuenta el papel que juegan los valores en el entorno en que se mueve el protagonista, y también cómo los valores de éste influyen en la gestación, diseño y formulación de una política.

En este sentido hay que buscar la interrelación de teoría - becho/fenómeno-valor.

Valores en el discurso

El periodo y los discursos generados en éste también son elementos que van generando valores.

Estos elementos contienen una carga ideológica nacional, regional o internacional. El discurso contiene los elementos que nos pueden ayudar a realizar el análisis en tanto que suma tanto la ideología como el entorno y los factores culturales.

Un ejemplo es el discurso que se da alrededor de la "Sociedad de la información”, el cual ha sido aceptado por la mayoría de los países. Lo mismo sucede con el desarrollo sustentable, sobre el que Amanda Spink realiza un análisis en su artículo "Information and a Sustainable future". 
Otro ejemplo puede ser Frank Webster, ${ }^{10}$ quien propone un análisis de esta sociedad a partir de perspectivas diversas:

- La tecnología.

- La economía.

- El mercado laboral.

- El espacio.

- La cultura.

En México se puede contrastar el discurso político con los hechos o programas de ejecución y de coordinación, por ejemplo.

Y también se pueden realizar comparaciones entre los países por su ideología, y sus niveles de gobierno, ya sean federales, estatales o municipales. Y también por el tipo de economía, ya sea de mercado libre o de intervención.

Análisis de valores en políticas de información

Esto lo realizan Overman y Cahill $^{11}$ a partir de los siguientes valores que identificaron:

a) Acceso a y libertad de información.

b) Privacidad.

c) Apertura a todos los que lo soliciten.

d) Utilidad de la información.

e) Costo beneficio.

f) Secreto y seguridad.

g) Pertenencia.

Cada uno de estos valores tiene una relación estrecha con la democracia, el derecho personal, el derecho a conocer y a saber, la ideología partidista o democrática, la necesidad de tener una autoridad burocrática y la propiedad intelectual.

Los valores pueden ser de índole restrictiva, como por ejemplo:

- utilidad,

- costo beneficio,

- secreto y seguridad,

- pertenencia y privacía,

10 Cfr. F. Webster. Theories of the information society. 2da ed. London: Routlege, 2002.302 p.

11 Cfr. E. S. Overman, y A. G. Cahill. "Information policy a study of value in the policy process" en Policy Studies Review 9 (4):803-818, 1990. 
o bien basarse en un aspecto distributivo, como:

- Acceso.

- Libertad.

- Apertura y privacía.

En una estructura normativa la privacidad considerada desde la perspectiva restrictiva desarrolla el tema de la protección mientras, que desde la perspectiva distributiva se aboca al acceso.

Análisis de los protagonistas

Es propuesto por Burgoyne ${ }^{12}$ y trata de identificar uno o más de los protagonistas que están inmersos en algunas políticas de información; recopila datos relativos a ellos, sus acciones, sus percepciones de la realidad, su conducta, su experiencia e historicidad y cómo y qué piensan acerca de un fenómeno o un hecho. Por protagonistas hay que entender actores y agentes participantes.

Esta metodología puede ser muy específica o muy libre. Este método de análisis implica una perspectiva cualitativa y para trazar una investigación utilizando esta metodología se necesita delimitar a los involucrados por su naturaleza, quiénes son ellos, cuáles son sus necesidades, qué es lo que desean, cuáles son sus percepciones, y cuáles sus conceptualizaciones.

\section{Naturaleza de la SOciedad}

Por su parte Tamara Eisenschitz ${ }^{13}$ señala que la información es transmitida en la sociedad y que el tipo de sociedad es un factor importante que nos ayudará a entender y enfocar nuestro estudio. El conocimiento en las sociedades puede ser considerado como subjetivo y objetivo a la vez, mientras que las interacciones en ellas son motivadas por consenso o disenso.

A partir de la naturaleza de la sociedad se pretenden analizar los asuntos de control y acceso a la información. Los tipos de sociedades que ésta enuncia son:

- una sociedad subjetiva cooperativa en donde el entendimiento de los fenómenos es de índole subjetivo y se mantiene la cooperación social,

12 Cfr. John G. Burgoyne. "Stakeholder analysis: en Cassel, C. y Simon G. Qualitative methods in organizational research. Sage Publication, 1994. p198-207.

13 Cfr. Tamara Eisenschitz. Information transfer policy: issues of control and access. Library Association, 1993. 175p. 
- una sociedad de cambios radicales donde impera la confrontación más que el consenso,

- una sociedad de cambios subjetivamente radicales donde es persistente el conflicto social y la subjetividad físicos y,

- una sociedad en donde se dan las interacciones sociales en diferentes niveles así como también los procesos y los esquemas de toma de decisiones.

Desde la perspectiva del funcionalismo, la teoría de sistemas sociales nos puede brindar explicaciones a nivel de interacción social sobre los procesos y esquemas de toma de decisiones.

Explicarse la naturaleza de la sociedad es de suma importancia para entender el sistema legal-jurídico, del cual emanan las regulaciones, las relaciones entre los agentes, la competitividad, las declaraciones gubernamentales en materia de información, los datos personales, los medios, la educación y la calidad.

\section{CARACTERÍSTICAS Y CAMPO DE ACCIÓN}

\section{Algunas características}

Sandra Braman ${ }^{14}$, en la década de los noventa, surgirió el análisis de las políticas de información a partir de sus características. Su propuesta es en el sentido de realizar el análisis según el sistema social o el sistema de gobierno (regime).

Se entiende por regime "un plan organizado que tiene un enfoque que converge en expectativas que son principios, normas, reglas o procedimientos en un área en particular". ${ }^{15}$

La información tiene las siguientes características:

- Reconocimiento Reciente: asunto de reciente discusión en la agenda de los procesos políticos. "Sabemos que el control y la manipulación de la información es una de las armas más poderosas del mundo moderno".

- Cantidad de protagonistas y tipos de protagonistas: tiene un gran número de protagonistas e incluye a aquellos que son de diferente tipo. La mayoría de los protagonistas proviene del sector privado.

- Tiene alcances y efectos sobre otras áreas. Las decisiones tomadas en el ámbito de política de información afecta áreas de otros asuntos.

14 Cfr. Sandra Braman. "The unique characteristics of information policy and their U:S: consequences", en V. P. Blake y R. Tjoumas, Information literacies for the twenty-first century. G.K. HALL\& CO, 1990. pp. 47-77. 
- No encaja en la manera tradicional de categorizar los asuntos de política; No se puede tipificar de una manera fácil en las áreas tradicionales.

- Existe un marcado grado de interdependencia entre las políticas de información en los diferente niveles de la estructura social. Las políticas de información efectuadas a diferentes niveles de la estructura social son interdependientes, reflejan contribuciones de nuevas formas de procesamiento de información, y se distribuyen y usan a partir de una expansión tecnológica, económica, social y política, y una interdependencia social.

Campo de acción.

Las políticas en sí pueden ser manifiestas, o visiblemente pertinentes a un área o asunto, o pueden ser latentes, si existen, pero no son visibles.

Algunas políticas pueden pertenecer a un área temática que ha sido creada por otra perspectiva, o por autores que abarcan la misma temática pero le dan otra denominación. Una política latente se desarrolla cuando hay fricciones entre los grupos en el gobierno, entre gobierno e iniciativa privada, o cuando participan intereses de la iniciativa privada.

Este contexto de políticas latentes y manifiestas ha producido varias perspectivas sobre políticas de información que en ocasiones tienen elementos similares; como ejemplo Pool, Dunn y Leeson nos presentan sus perspectivas en el siguiente cuadro.

\begin{tabular}{|l|l|l|}
\hline \multicolumn{3}{|c|}{ Políticas de información } \\
\hline \multicolumn{1}{|c|}{ Pool,1983 } & \multicolumn{1}{|c|}{ Dunn, 1982 } & \multicolumn{1}{c|}{ Leeson, 1984 } \\
\hline Disponibilidad de recursos & Creación & Conocimiento técnico y difusión \\
\hline Organización de los accesos & Distribución y comunicación & Componente físico \\
\hline $\begin{array}{l}\text { Regulación y detección de } \\
\text { los problemas }\end{array}$ & $\begin{array}{l}\text { Almacenamiento y recuper- } \\
\text { ación }\end{array}$ & $\begin{array}{l}\text { Estructura de facilidades y } \\
\text { redes }\end{array}$ \\
\hline & Uso y aplicación & $\begin{array}{l}\text { Servicios en que terminó, } \\
\text { condiciones y uso de facilidades } \\
\text { de redes }\end{array}$ \\
\hline & & $\begin{array}{l}\text { Tratamiento de la información } \\
\text { en sí mismo }\end{array}$ \\
\hline
\end{tabular}

También las políticas de información son vistas desde el contexto económico. Mientras que otros autores las perciben como el impacto, las obligaciones y las relaciones que tienen suscritas con el propio sector económico, en el ámbito de las telecomunicaciones las políticas de información giran alrededor de la infraestructura.

Otro planteamiento se hace a partir de la producción de la información, la cual abarca: 
- La creación de la información: la creación, la generación y el agrupamiento.

- El procesamiento: la parte cognitiva que realiza el humano y la algorítmica que se hace a través de modelos matemático/computacionales.

- Transportación.

- Distribución.

- Almacenamiento.

- Destrucción.

- Recuperación.

Una aproximación sobre cómo hacer que el estudio sea relevante para los tomadores de decisiones se enmarca en los siguientes problemas:

1. Conceptuales: debido a tantas definiciones que existen sobre políticas de información.

2. De toma de decisiones: la información no es vista como un recurso estratégico.

3. Estructurales.

\section{ANÁLISIS DE POLÍTICAS}

Charles Lindblom ${ }^{16}$ nos remite a un análisis de política pública y nos recomienda una posible metodología a seguir:

- Estudiar cómo aparecen los problemas.

- Recoger éstas en una agenda de decisiones del gobierno.

- Averiguar cómo proceden los hacedores de políticas.

- Averiguar cómo plantean los ciudadanos las acciones.

Lindblom argumenta que para "entender la elaboración de políticas públicas hay que comprender la vida y la actividad política" ${ }^{17}$

Debe quedar de manifiesto que hay que realizar y hacer más "efectivas las políticas para resolver problemas sociales y cómo hacer una política que responda al control popular". 18 A la vez hay que concentrarse en "el conflicto entre la razón y el análisis y la ciencia por un lado, y la política y la democracia por el otro". ${ }^{19}$

16 Cfr. C. Lindblom. El proceso de elaboración de políticas públicas. México: M.A. Porrúa: Madrid: INAP, 1991.

17 C. Lindblom. Op cit. p.16. 
Para la elaboración de políticas hay que considerar:

- La posibilidad de error o fallo en el análisis.

- Los conflictos de valores que pueden surgir.

- El tiempo y el costo.

- El problema tal como lo vamos a seleccionar.

Lindblom aboga por un análisis científico donde el analista de las políticas "formula el problema como un todo, especifica los objetivos y valores, solicita y evalúa las soluciones alternativas e identifica la solución que se corresponde mejor con los valores formulados". ${ }^{20}$

Para hacer lo anterior hay que tomar en cuenta:

1. El ideal estratégico.

2. Los acuerdos prácticos comúnmente basados en la naturaleza periódica y en la ideología.

3. Las normas de la sociedad y/o la comunidad.

4. El control: la persuasión, las amenazas y el intercambio.

5. La eficiencia de la autoridad en el intercambio.

6. El control mutuo y el ajuste.

\section{INVESTIGACIÓN EN POLIITICA}

Ann Majchrzak ${ }^{21}$ presenta una discusión sobre la naturaleza de la investigación en políticas (policy research). Define la investigación en políticas como

"el proceso de conducir la investigación, y el análisis de aspectos fundamentales o problemas sociales para proveer a los tomadores de decisiones con recomendaciones pragmáticas, orientadas hacia acciones que mitigen los problemas".

Visto como un proceso "provee opciones e información a los tomadores de decisión para resolver los problemas que tienen". ${ }^{22}$ En otras palabras, se trata de "una secuencia de actividades que empieza con la preparación del estudio y termina con un informe breve y algunas recomendaciones". ${ }^{23}$

Cualquier política empieza con un problema de índole social, atraviesa por un proceso de investigación que propone acciones para mitigar los problemas,

20 Op cit. p. 23

21 Cfr. Ana Majchrzak. Methods for policy research. Newbury Park: Sage publications,1984

22 Ana Majchrzak. Op cit.p.12.

23 Op cit.p. 11. 
y piensa y desarrolla comunicados para los tomadores de decisión de políticas. Estos estudios pueden ser divididos en:

- Investigación básica: se realiza principalmente en las universidades y la academia con base en el consenso y la concordancia.

- Investigación técnica: son proyectos estructurados para resolver problemas específicos.

- Análisis de políticas: es un estudio sobre el proceso decisorio de las políticas.

- Investigación de políticas: está compuesta tanto por la investigación básica como por el análisis de política cuya finalidad es resolver un problema social.

Ante este panorama cabe preguntarse ¿qué pasa con la investigación sobre políticas de información? y lo que tenemos que averiguar es:

1. ¿Dónde está puesto el enfoque: en el problema o en la solución?

2. ¿De dónde provienen los fondos?

3. ¿Cómo es la estructura organizacional de la institución?

4. ¿Cuál es el área disciplinaria del investigador?

Otros elementos que influyen en los estudios de la "investigación en políticas" son:

- El enfoque multidimensional.

- La orientación empírico-inductiva.

- La incorporación explícita de valores.

- El hecho de que abarca el presente y el futuro.

- El hecho de que mucho depende del enfoque del investigador.

\section{ANÁLisis DEL ALCANCE E INTENCIÓN DE LOS ESTUdiOS}

Eileen Trauth ${ }^{24}$ propone un método para ubicar en un mapa bidimensional los tipos de estudios realizados en Estados Unidos en torno a las políticas de información. Los estudios de índole descriptiva-prescriptiva los ubica en el plano horizontal y en el plano vertical integrador -particular.

Los estudios descriptivos: describen las políticas y cómo debe ser su implantación.

Los estudios prescriptivos tratan las propuestas hechas por los protagonistas y que podrían influir en la formulación de políticas. 
Los estudios integradores comprenden los hechos de manera interdisciplinaria y pueden ser vistos desde diferentes perspectivas disciplinarias.

$\mathrm{Y}$ los estudios particulares son los que han sido solicitados para un único propósito sobre un tema en particular.

\section{Contribución Descriptiva}

Charles McClure, Moen y Bertot ${ }^{25}$ proponen un abanico de técnicas que presentan un panorama multidimensional en relación con una propuesta de políticas de información. Estos autores proponen la revisión de los documentos utilizando:

- El contexto histórico.

- La revisión y selección de los documentos claves en el ámbito de la políticas de información, como son los planes, programas y proyectos que nos pueden acercar al panorama de las acciones emprendidas.

- Los modelos descriptivos; hay que utilizar la técnica de representación grafica a partir de dos variables o más.

- El análisis “codo a codo”, una conocida técnica analítico-descriptiva que permite realizar comparaciones descriptivas y aislar con base en un conjunto de parámetros.

- La revisión de instrumentos relacionados con la política: la cual nos brinda una mejor y detallada identificación y discusión de los documentos. Esta revisión debe identificar el grado de selección, comparar estas herramientas y discutir los elementos claves.

- La revisión de la literatura, método tradicional de ubicar las perspectivas y los contextos de las políticas.

- La identificación y descripción de los asuntos claves que resuman el estado actual de un área en particular.

\section{TAXONOMÍA DE POLÍTICAS DE INFORMACIÓN}

Terrence Maxwell ${ }^{26}$ propone un análisis de contenido para extraer los componentes claves de los documentos y posteriormente utilizar un modelo que

25 Cfr. C. R. McClure; W. E. Moen y J. C. Bertot. "Descriptive assessment of information policy iniciatives: the government information locator service (GILS) as an example", en Journal of the American Society for Information Science, 50 (4):314-330, 1999.

26 Cfr. Terrence Maxwell. "Mapping information policy frames: The politics of the digital millennium copyright act", en Journal of the American Society for Information Science and Technology 55 (1): 3-12, 2004. 
vincule los datos que se están utilizando y el fenómeno que se esté estudiando. Esto nos lleva a una relación protagonista entre los documentos generados por ellos, el contenido de tales documentos y las acciones propuestas, más sus respectivos resultados.

\section{A MANERA DE DISCUSIÓN}

Los métodos de recolección de datos propuestos y descritos con anterioridad nos permiten visualizar el amplio espectro de la investigación existente en materia de "políticas de información".

Los veinte largos años que van de 1984 (Trauth) al 2004 (Maxwell), no nos permiten aseverar que son éstos los únicos métodos para obtener datos y así fortalecer los estudios en esta temática. Sin embargo cada una de las propuestas descritas por Braman (1990, 1993, 1995), Browne (1997), Burgoyne (1994), Eisenschitz (1993, 1998), Lindblom (1968,1991), Marjchrzak (1984), Maxwell (2004), McClure, Moen, Bertot (1999), Overman y Cahill (1990), Rowlands $(1996,1998,2003)$ y Trauth (1986), presentan diferente estrategias para la obtención de datos.

Hasta el momento no ha habido alguna noticia que evidencie la aplicación de tales propuestas por parte de otros pares.

Esto no indica que debemos descartarlas, más bien nos indica que hay que analizarlas y aplicarlas de manera sistemática con el fin de recopilar datos útiles, que a su vez se conviertan en información que permita tener un panorama cada vez más claro del quehacer sobre la investigación en el área de políticas de información.

Siguiendo la perspectiva de Glaser y Strauss y su propuesta del método "teoría fundada en datos" que "...es una estrategia para manejar datos de una investigación proveyendo modos de conceptualización para describir y explicar fenómenos", ${ }^{27}$ podemos proponer metodologías más concretas para efectuar investigación sobre políticas de información. Cada investigación se apoya tanto en los datos obtenidos por la investigación en curso, como en aquellos obtenidos por otras investigaciones que hayan empleado la misma metodología.

Paulatinamente estos datos obtenidos de manera sistemática nos servirán para realizar predicciones, explicaciones, interpretaciones y hacer aplicaciones, y nos ayudarán a generar conocimientos más profundos sobre la temática estudiada.

A la vez nos harán más perseverantes para descubrir elementos que permitan la gestación de un enfoque teórico si no en toda el área sí al menos en una de sus partes. 
La tarea no será fácil ya que hay que recorrer un camino arduo para seguir probando los métodos propuestos y realizar comparaciones entre los datos obtenidos y las hipótesis propuestas.

Glaser y Strauss sugieren que nos apeguemos a los siguientes lineamientos, en donde la formulación:

- Debe facilitar la predicción y explicación de conductas.

- Debe ser de utilidad para el progreso de la investigación teórica del área.

- Debe ser reproducible tanto en su aplicación como en la praxis; es decir, proveer a los estudiosos del área elementos para entender la situación y elementos de control.

- Debe proporcionar perspectivas sobre algunas conductas y una posición intelectual hacia los datos.

- Debe ser una guía que brinde un estilo para realizar una investigación sobre un área temática.

Expresado de otra manera el método "teoría fundada en datos"(grounded theory) puede considerarse como guía en el proceso de recopilación y análisis de datos.

Al utilizar cualquiera de los métodos descritos se podría integrar un modelo de análisis que nos guiará para realizar comparaciones entre los datos obtenidos ya sea por observación o acciones, o que sean descritos en documentos o entrevistas.

Eso no significa que hay que descartar teorías afines al tema ni dejar de transmitir ese conocimiento de teorías ya consolidadas. Más bien hay que transmitir también las teorías de alcance medio y los enfoques teóricos que nos presentan un marco para la recopilación de datos de manera sistemática. Lo cual ha de seguirse con la manera de realizar la interpretación de tales teorías de tal manera que aporten elementos para un posible apuntalamiento de un probable enfoque teórico-metodológico.

En suma, en estos momentos la búsqueda de una metodología propia para los estudios de la información sobre la temática "políticas de información”, debería ser un nicho por desarrollarse en el entorno de la investigación referida a la bibliotecología y los estudios de la información.

Considerando lo anterior la aplicación de cualesquiera de los métodos de recopilación de datos propuestos ayudará a tener al menos una idea de estructura lógica, a identificar algunos de los elementos claves que intervienen en la política de información y, posteriormente, a realizar el análisis y el de sus respectivas características así como a identificar las posibles relaciones entre los elementos con el fin de reducir la complejidad. 


\section{BibLIOGRAFÍA}

Bender, D.R. A strategy for international information policy, en LIBRI 43 (3): 210-231, 1993.

Braman, Sandra. The unique characteristics of information policy and their U:S: consequences, en Blake, V.P. y Tjoumas R. Information literacies for the twenty-first century. G.K. HALL\& CO, 1990. pp. 47-77.

- - Harmonization of systems: the third stage of the information society, en Journal of Communication. 43 (3): 133-140, 1993.

- - Horizon of the state. Information policy and power, en Journal of Communication 45 (4): 4-24, 1995.

Browne, Mairead. The field of information policy. 1. Fundamental concepts, en Journal of Information Science 23 (4):261-275, 1997.

- - The field of information policy. 2. Redefining the boundaries and methodologies, en Journal of Information Science 23 (5):339-351, 1997.

Burgoyne, John G. Stakeholder analysis: En Cassel, C. y Simon G. Qualitative methods in organizational research. Sage Publication, 1994. p198-207.

Chartrand, R.J. Legislating information policy, en Bulletin of the American Society for Information Science 12 (5):10, 1986.

Eisenschitz., Tamara. Information transfer policy: issues of control and access. Library Association, 1993. 175p.

- - Internet law and information policy. ASLIB Proceedings 50 (9):267-273, 1998.

Entman, R. Framing: toward clarification of a fractured paradigm, en Journal of Communication 43 (4):51-58, 1993.

Glaser, B. y Strauss, A. The discovery of grounded theory: strategy or qualitative research. Aldine, $1967.271 \mathrm{p}$

Hernon, Peter. A research agenda beyond 2000, en Library $\mathcal{E}$ information Science Research 19 (3): 209-216, 1997.

- - Going beyond "same old, same old, en Journal of Academic Librarianship 23 (3): 169-176, 1997.

- - The literature on government information policies, practices and services in the coming years: topics meriting inclusion, en Government Information Quarterly 14 (3):221-228, 1998.

- - Components of research process, en Journal of Academic Librarianship 27 (2):81-89, 2001.

Lindblom, C. El proceso de elaboración de politicas públicas. México : M.A. Porrúa : Madrid : INAP, 1991. 160p.

Liu, Yin Quan, The impact of national policy on developing information infrastructure nationwide issues in P.R. China and the U.S., en LIBRI 46 (4):175-183, 1996.

Majchrzak, Ann. Methods for policy research. Newbury Park: Sage publications,1984.111p. 
Maxwell, Terrence. Mapping information policy frames: The politics of the digital millennium copyright act., en Journal of the American Society for Information Science and Technology 55 (1): 3-12, 2004.

McClure, C.R., Moen, W.E. y Bertot, J.C. Descriptive assessment of information policy iniciatives: the government information locator service (GILS) as an example, en Journal of the American Society for Information Science 50 (4):314-330, 1999.

Milevski, S. Information policy through public laws of the 95th-the 98th Congresses, en Proceedings of the American Society for Information Science Annual meeting, 23: 211-219, 1986.

Overman, E.S. y Cahill, A.G. Information policy a study of value in the policy process, en Policy Studies Review 9 (4):803-818, 1990.

Rowlands, Ian. Understanding information policy: concepts, frameworks and research tools, en Journal of Information Science 22 (1):13-25, 1996.

- - Some compass bearing for information policy orienteering, en ASLIB Proceedings 50 (8):230-237, 1998.

- - Knowledge production, consumption and impact: policy indicators for a changing world. en ASLIB Proceedings 55 (1/2): 5-12, 2003.

Rist. R.C. Influencing the policy process with qualitative research, en Denzin, N.K. y Lincoln, Y.S. Handbook of qualitative research. Sage, 1994. p.545-557.

Spink, A. Information and a sustainable future. LIBRI 45 (3/4): 203 208, 1995.

Trauth, Eileen. An integrative approach to information policy research, en Telecommunication Policy 10 (1): 41-50. 1986.

Webster, F. Theories of the information society. $2 \mathrm{da}$ ed. London: Routlege, 2002.302p 